\title{
Sporothrix brasiliensis and interaction Acanthamoeba castellanii
}

Tavares, P.L., Universidade Federal do Rio Grande do Sul, Porto Alegre, Brazil.

Heidrich, D., Universidade Federal do Rio Grande do Sul, Porto Alegre, Brazil. Ribeiro, A.C., Universidade Federal do Rio Grande do Sul, Porto Alegre, Brazil. Rott, M.B., Universidade Federal do Rio Grande do Sul, Porto Alegre, Brazil. Scroferneker, M.L., Universidade Federal do Rio Grande do Sul, Porto Alegre, Brazil (Presenting author).

\section{Introduction}

Sporothrix brasiliensis is a dimorphic fungus found in the environment, vegetation and wood, being the etiological agent that causes human and feline sporotrichosis. Sporotrichosis has been identified in several areas of the world; most cases come from Central and South America mainly in Mexico, Colombia, Brazil and Peru, as well as the African continent. The genus Acanthamoeba is a group distributed in soil, water and air. Some species may be pathogenic and opportunistic, they feed on a variety of microorganisms, but some of them have evolved becoming resistant since they are able to survive and leave the amoebae after internalization. The A. castellanii presents two stages of life, the trophozoite and the cyst. In the trophozoite state the amoeba fed affectively, the encystment occurs as a form of protection to unfavorable conditions amoeba.

\section{Objectives:}

to analyze the interaction of Sporothrix brasiliensis in the process of encystment of Acanthamoeba castellani.

\section{Methods:}

the trophozoites $\left(10^{5}\right.$ cells $\left.\mathrm{mL}-1\right)$ were seeded onto the culture plates using Tris buffer as a encystment inducer. After 30 minutes of acclimatization, the amoebas were incubated with conidia of $S$. brasiliensis in a ratio of $1: 1$ at $30^{\circ} \mathrm{C}$ for $96 \mathrm{~h}$. The control was isolated amoebae in the medium of encystment. At the end of the incubation time, the number of trophozoites and cysts were determined in the Fuchs-Rosenthal counting chamber. The experiment was performed in triplicate and repeated once. The data will be expressed as percentage of encystment (number of cysts by total number of amoebas).

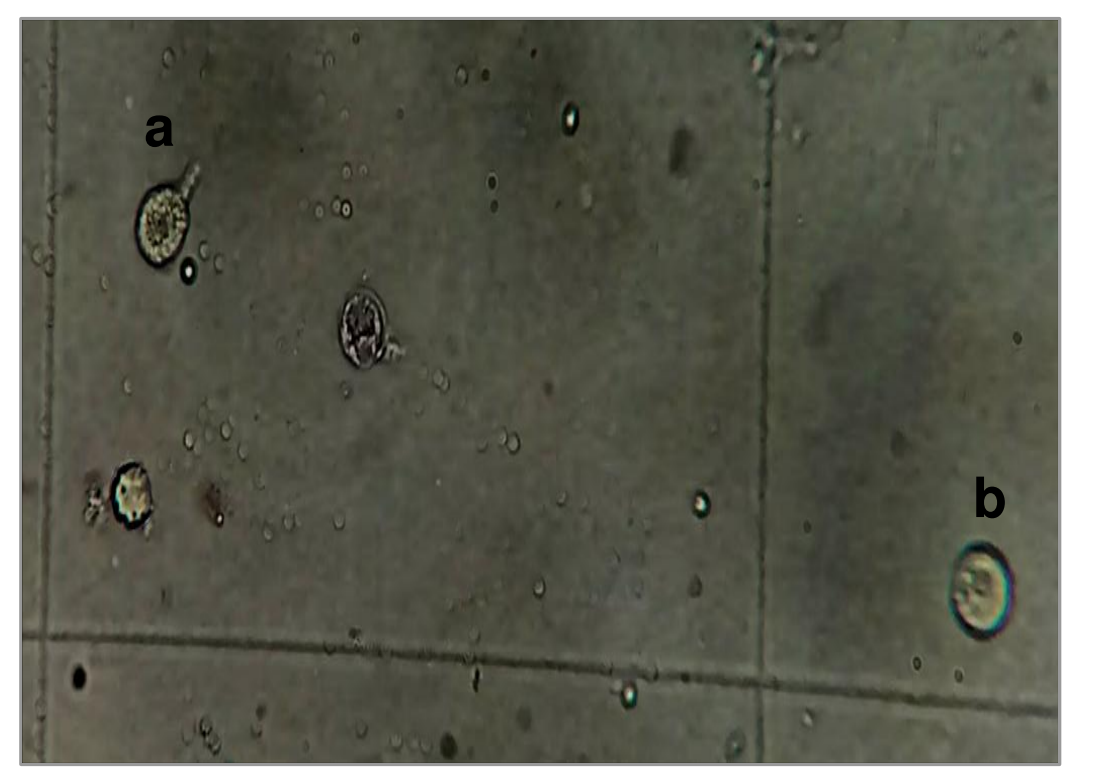

Figure 1. Sporothrix brasiliensis and interaction Acanthamoeba castellanii. a internalized fungus coming out of amoeba; b amoeba in process of encystment .

Results:

after the amoeba contact with the conidia of $S$. brasiliensis cysts were formed in $74.5 \%$ and remained as trophozoites $25.5 \%$. The control sample showed $54 \%$ of $A$. castellanii cysts and $46 \%$ of trophozoites. Demonstrating a $20.5 \%$ increase in cysts formation between the samples with the presence of the fungus compared to amoeba without the fungus in the solution of encystment.

\begin{tabular}{lrr}
\hline & \multicolumn{2}{r}{ Encystment (\%) } \\
\cline { 2 - 3 } & Cysts & Trophozoite \\
A. castellanii and S. brasiliensis & $74.50 \%$ & $25.50 \%$ \\
A. castellanii & $54 \%$ & $46 \%$ \\
\hline
\end{tabular}

Conclusion:

the interaction between the fungus and the amoeba resulted in an increase in the population of cysts and in the decrease of the trophozoite form. 